\title{
Best management of irritable bowel syndrome
}

\author{
Christopher J Black (D) , ${ }^{1,2}$ Alexander Charles Ford (i) 1,2
}

${ }^{1}$ Leeds Gastroenterology Institute, St. James's University Hospital, Leeds, UK ${ }^{2}$ Leeds Institute of Medical Research at St. James's, University of Leeds, Leeds, United Kingdom

\section{Correspondence to}

Professor Alexander Charles Ford, Leeds Gastroenterology Institute, St. James's University Hospital, Leeds LS9 7TF, UK; alexf12399@ yahoo.com

Received 8 April 2020 Revised 2 May 2020 Accepted 5 May 2020 Published Online First 28 May 2020

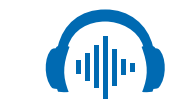

Listen to Podcast fg.bmj.com

\section{Check for updates}

(C) Author(s) (or their employer(s)) 2021. No commercial re-use. See rights and permissions. Published by BMJ.

To cite: Black CJ, Ford AC. Frontline Gastroenterology 2021;12:303-315.

\section{ABSTRACT}

Irritable bowel syndrome (IBS) is a common disorder of gut-brain interaction which can have a considerable impact on quality of life. Following diagnosis, timely and evidence-based management is vital to the care of patients with IBS, aiming to improve outcomes, and enhance patient satisfaction. Good communication is paramount, and clinicians should provide a clear explanation about the disorder, with a focus on exploring the patient's own beliefs about IBS, and a discussion of any concerns they may have. It should be emphasised that symptoms are often chronic, and that treatment, while aiming to improve symptoms, may not relieve them completely. Initial management should include simple lifestyle and dietary advice, discussion of the possible benefit of some probiotics, and, if this is unsuccessful, patients can be referred to a dietician for consideration of a low FODMAP (fermentable oligosaccharides, disaccharides, monosaccharides and polyols) diet. Antispasmodics and peppermint oil can be used first-line for the treatment of abdominal pain. If patients fail to respond, central neuromodulators can be used second-line; tricyclic antidepressants should be preferred. Loperamide and laxatives can be used firstline for treating diarrhoea and constipation, respectively. Patients with constipation who fail to respond to laxatives should be offered a trial of linaclotide. For patients with diarrhoea, the 5-hydroxytryptamine- ${ }_{3}$ receptor agonists alosetron and ramosetron appear to be the most effective second-line drugs. Where these are unavailable, ondansetron is a reasonable alternative. If medical treatment is unsuccessful, patients should be referred for psychological therapy, where available, if they are amenable to this. Cognitive behavioural therapy and gutdirected hypnotherapy are the psychological therapies with the largest evidence base.

\section{INTRODUCTION}

Irritable bowel syndrome (IBS) is one of the most common disorders of gut-brain interaction, with a population prevalence of between $5 \%$ and $10 \% .^{12}$ The cardinal symptoms are abdominal pain, related to defaecation, associated with a change in the frequency or form of stools. ${ }^{3}$ Symptoms are chronic, often severe and have a substantial impact on quality of life. ${ }^{4}$ More than $80 \%$ of patients will consult their general practitioner (GP), ${ }^{5}$ and the majority are managed successfully in this setting. ${ }^{6}$ However, some patients, particularly those who fail to respond to first-line treatment, or those in whom there is any diagnostic uncertainty, for example, older patients in whom underlying organic pathology may need to be excluded, will be referred to secondary care. Managing patients with IBS accounts for approximately $25 \%$ of a gastroenterologist's time in the outpatient clinic, ${ }^{7}$ and the associated costs of investigation and treatment are considerable. ${ }^{8}$

When reviewing any patient with IBS, the first consideration should be the requirement for any further investigation. We have previously written about the importance of adopting a rational approach, which prioritises making a diagnosis on clinical grounds in the majority of cases, following a limited number of essential investigations, for example, routine bloods, including coeliac serology. ${ }^{9}$ Exhaustive investigation is not only undesirable, but unnecessary, as tests are frequently normal, and this is likely to make patients feel that the cause of their symptoms has been missed, rather than providing reassurance. ${ }^{10}$ Indeed, taking a rational approach to investigation, and making an early diagnosis, are key components of recommendations calling for physicians to standardise the care of patients with IBS, with the aim of improving the quality of care overall, and enhancing patient satisfaction. ${ }^{11}$ The next step is to start treatment in a timely fashion, ideally during the first consultation if the diagnosis is secure and the 


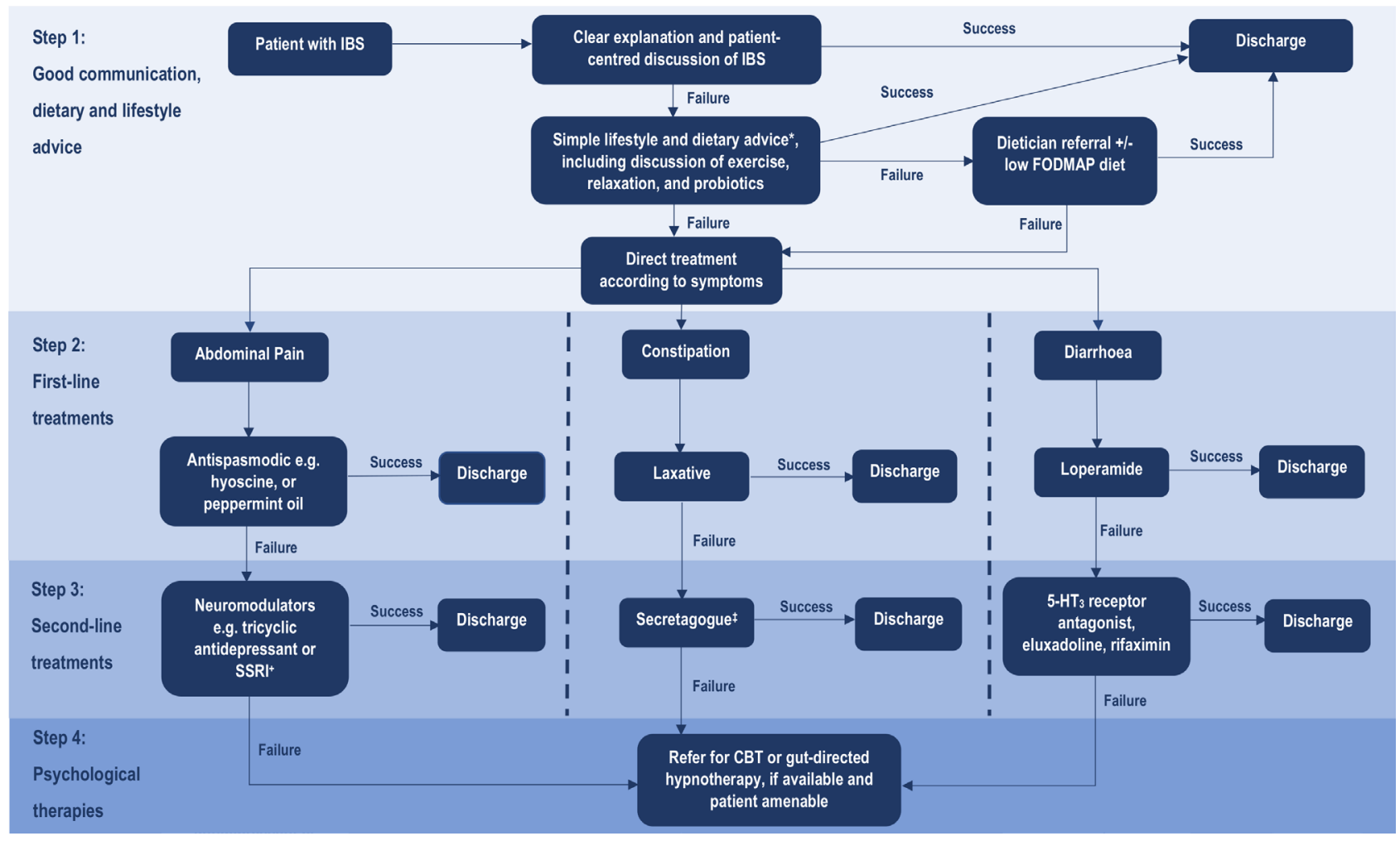

Figure 1 Suggested algorithm for the management of irritable bowel syndrome. CBT,cognitive behavioural therapy; FODMAP, fermentable oligosaccharides, disaccharides, monosaccharides and polyols; IBS, irritable bowel syndrome; SSRI, selective serotonin reuptake inhibitor; TCA, tricyclic antidepressant; 5- $\mathrm{HT}_{3}$, 5-hydroxytryptamine- ${ }_{3}$. ${ }^{*}$ As per NICE IBS dietary advice sheet, plus consider ispaghula husk. + Tricyclic antidepressants should be first choice, starting at a dose of $10 \mathrm{mg}$ at night, and titrating slowly (e.g. by $10 \mathrm{mg}$ per week) according to response and tolerability. Continue for at least 6 months if patient reports symptomatic response. ₹Review efficacy after 3 months of treatment, and discontinue if no response.

relevant limited diagnostic tests to exclude another condition have already been performed in primary care. However, there is a recognition that confidence in managing IBS will vary between physicians, dependent on expertise.

In general, treatment is targeted at addressing a patient's most troublesome symptoms, be that abdominal pain, diarrhoea, constipation or bloating. Although several factors have been implicated in the pathophysiology of IBS, including the gut-brain axis, alterations in the microbiome, genetic factors and visceral hypersensitivity, there is currently no role for using these to guide therapy in routine practice. Moreover, it is likely that, even among patients with the same symptoms, the underlying pathophysiology responsible for them will vary. Consequently, although treatments may be designed to address theoretical pathophysiological abnormalities, there is no way to assess response through objective measurement of these and, instead, the clinician must rely on patient-reported symptom response to determine treatment success.

This review aims to provide physicians with a practical, comprehensive and evidence-based framework for treating IBS (figure 1). It will focus on the importance of good communication, the role of dietary and lifestyle advice and second-line dietary strategies.
First-line and second-line drug treatments will be evaluated, as will newer second-line treatments targeting abnormal stool form specifically. Finally, the place of psychological therapies in IBS will also be discussed. One problem with randomised controlled trials (RCTs) of therapies is that, in most IBS treatment trials, active drugs are compared with a placebo, rather than with each other. Therefore, the absence of head-to-head treatment comparisons makes it difficult to understand the relative efficacy of drugs, which is important when doctors and patients are choosing treatments. Network meta-analysis can circumvent this problem, to some extent, by providing the likelihood of which treatment is likely to be the most efficacious in each clinical scenario, and the findings from these types of evidence synthesis will be discussed, where available (table 1).

\section{THE IMPORTANCE OF GOOD COMMUNICATION}

Before embarking on the prescription of any treatment, it is vital to recognise the role that good communication plays in management. An online survey of people with IBS revealed that many had a negative view of their relationship with healthcare professionals, with concerns about not being heard and a lack of empathy. ${ }^{12}$ Indeed, patients report a sense of frustration and 


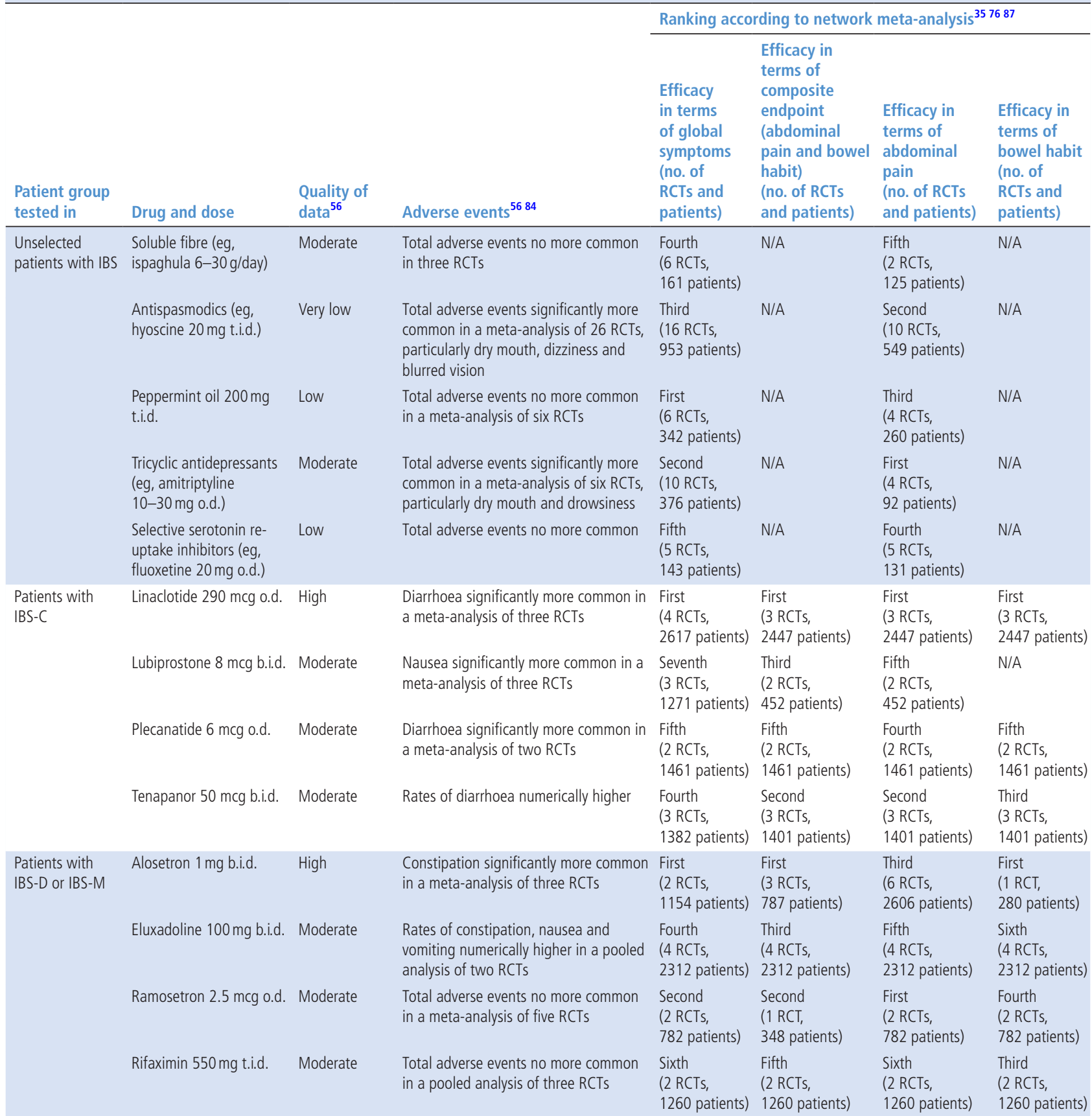

b.i.d., two times per day; IBS, irritable bowel syndrome; IBS-C, IBS with constipation; IBS-D, IBS with diarrhoea; IBS-M, IBS mixed; o.d., once daily; RCTs, randomised controlled trials; t.i.d., three times per day.

isolation, reporting that consultation with medical experts rarely clarified their understanding of IBS or improved their management. ${ }^{13}$ This might in part reflect unrealistic expectations of patients, many of whom report a willingness to try any treatment in their desperation for a cure, only to be left disappointed when symptoms are not relieved completely. ${ }^{14}$ However, it also reflects a mismatch between patients' ideal expectations of a consultation, and the reality of their experiences. In one survey of over 1000 patients, more than $90 \%$ wanted their doctor to give comprehensive information about IBS and provide sources for additional information, to listen well and answer questions, and to provide information about medication. Unfortunately, in recalling their prior experiences of healthcare, only $40 \%$ felt that their doctor provided information, $64 \%$ felt they had been listened to and 47\% felt supported.

Equally, many patients have significant misconceptions regarding the nature of IBS and the prognosis. In one questionnaire study of over 250 patients with IBS, less than one-third knew abdominal pain was a key symptom, $40 \%$ thought colonoscopy could diagnose IBS, 30\% believed IBS increased the risk of developing 
inflammatory bowel disease and one in seven believed that IBS could lead to cancer. ${ }^{15}$ There are also issues with doctors' perceptions of IBS; the majority of GPs in one study believed it was primarily a psychological disorder, ${ }^{16}$ or in another survey a response to stress. ${ }^{17}$ Moreover, a qualitative study revealed that many doctors hold two contrasting views of IBS, the first being a publicly expressed 'medical' definition, and the second being a private view, incorporating their own experiences of managing patients and absorbed prejudices. $^{18}$

It is therefore clear that there is a mismatch between views of doctors and patients regarding IBS, which may limit the usefulness of the patient-clinician interaction. A recent systematic review identified five practices that help foster a more positive, meaningful and engaged consultation. ${ }^{19}$ First, doctors should prepare with intention, taking a moment to focus before greeting a patient. Second, they should listen intently and completely. Third, they should explore what the patient cares about, and agree on what matters most, setting priorities in partnership. Fourth, they should seek to connect with the patient's story, considering life experiences that influence their health, as well as acknowledging positive action and success. Finally, doctors should explore the patient's emotions, taking note of any emotional cues.

Adopting this holistic approach has the potential to improve outcomes for patients with IBS, ${ }^{20}$ ensuring that their ideas and concerns are elicited. It is also vital to provide a clear explanation about the nature of IBS as a functional disorder and what this means, including why investigations have been normal, and that this is expected. Patient expectations should also be managed appropriately with discussion focussing on the prognosis of IBS, explaining that around twothirds of patients experience chronic symptoms, ${ }^{21}$ with treatment targeted at improving symptoms, rather than complete symptom relief. Finally, doctors should outline treatment options, including the role of second-line treatment if initial management strategies prove unsuccessful. All of this will take more time than the standard allotted to a new or follow-up appointment in clinic. In our specialist clinic we allow $30 \mathrm{~min}$ for a new patient consultation, and $15 \mathrm{~min}$ for a follow-up. We usually allow patients a minimum of two follow-up visits, before returning them to the care of their GP, in order to allow commencement of treatments not available in primary care, with assessment of their efficacy.

\section{GENERAL DIETARY AND LIFESTYLE ADVICE}

A discussion of simple dietary and lifestyle advice should be part of the care of all patients with IBS. The concept of self-help is important in empowering patients to take control of managing their condition.

\section{Diet}

Patients with IBS frequently report that symptoms are associated with eating certain foods. ${ }^{22} 23$ Consequently, many patients will exclude these from their diet with the aim of improving symptoms. ${ }^{24}$ However, should they report a positive response, this is more likely to reflect the fact that, to some degree at least, symptoms are expected to be meal-related, as per diagnostic criteria, rather than reflecting a true food allergy, mediated via an immune response.

Patients may seek to identify perceived food intolerances using bloods tests, although there is currently insufficient evidence to support this approach. In one RCT, 150 patients were randomised to either a 12-week diet excluding foods to which they showed cross-reactivity on IgG antibody testing, or to a sham diet, where they excluded the same number of foods to which they had tested positive, but not the specific foods to which they reacted. ${ }^{25}$ A greater proportion of patients following the true exclusion diet reported symptom improvement, but this was not statistically significant. In another study, leucocyte activation testing of peripheral blood samples was conducted to identify possible food intolerance, and patients were randomised to a true versus sham elimination diet. ${ }^{26}$ Participants following a true elimination diet had a significantly greater improvement in symptom scores, compared with those allocated to a sham diet. However, there was no significant difference in the proportion of patients reporting adequate relief of IBS symptoms, nor in quality of life measures. More recently, one study suggested that people with IBS may have atypical food allergies, which are not mediated via classical $\operatorname{IgE}$ pathways, although this requires corroboration. ${ }^{27}$ Nevertheless, and irrespective of testing, empirical dietary management represents an important first-line treatment strategy.

The National Institute for Health and Care Excellence (NICE) endorse a food fact sheet produced by the British Dietetic Association, providing patients with clear and concise dietary advice. ${ }^{28}$ This gives general recommendations, emphasising the importance of eating regular meals, limiting alcohol and caffeine intake, maintaining adequate hydration and reducing processed food consumption. There is also advice relating to specific symptoms. For example, patients with flatulence and bloating are recommended to limit intake of gas-producing food like beans and pulses, and are informed of the potentially beneficial effects of eating linseeds. However, the latter is based primarily on anecdotal observations. A 4-week RCT in 40 patients failed to show a benefit for either whole or ground linseeds over normal diet in terms of improvement in IBS symptom severity, or individual symptoms, including bloating. ${ }^{29}$ Patients with diarrhoea are cautioned to avoid sugar-free sweets, mints, gum and soft drinks that contain sorbitol, mannitol or xylitol. Advice is also given regarding dietary fibre, which is 
discussed in greater detail below, with an increased intake recommended for those with constipation, but a reduction in patients with diarrhoea.

\section{Fibre}

The role of dietary fibre in treating IBS was first examined over 40 years ago. Bran is an example of insoluble fibre, which undergoes little physical change as it passes through the gut, bulking stools and increasing stool water content, with the potential to accelerate gastrointestinal transit times. ${ }^{30}$ However, fibre may also be soluble in water, such as ispaghula, forming a gel that interacts with gut bacteria, resulting in production of metabolites, including short-chain fatty acids and secondary bile acids. ${ }^{31}$ These metabolites may, in turn, stimulate gastrointestinal transit, possibly through effects on enteric nerves and smooth muscle, or play a role in immune-mediated anti-inflammatory pathways. ${ }^{32}$

A systematic review and meta-analysis published in 2014 examined effect of dietary fibre supplementation on IBS symptoms. ${ }^{33}$ Overall, there was a significant benefit of fibre on global symptoms (relative risk (RR) of symptoms persisting $=0.86 ; 95 \%$ CI 0.80 to 0.94 ) in 14 RCTs, containing 906 patients. However, subgroup analysis demonstrated that benefit was confined to RCTs of ispaghula ( $R R=0.83 ; 95 \% \mathrm{CI} 0.73$ to 0.94 ), with no evidence for bran $(\mathrm{RR}=0.90 ; 95 \% \mathrm{CI} 0.79$ to 1.03). Fibre can exacerbate pain, bloating and flatulence, and, although the meta-analysis found there were insufficient data to enable analysis of adverse events according to fibre type, these side effects are generally considered to be a greater issue for insoluble fibre. $^{34}$

In a network meta-analysis of 'traditional' therapies for treating IBS, which also included antispasmodics, peppermint oil and central neuromodulators, ispaghula was ranked fourth for effect on global symptoms and fifth for abdominal pain. ${ }^{35}$ Consequently, other first-line treatment strategies might be more efficacious, although, as a 'natural' treatment, ispaghula may appeal to some patients who are reluctant to try drugs. Due to its ability to improve stool viscosity and frequency, it is perhaps deployed most logically for treating IBS with constipation (IBS-C), although the evidence for this is inconclusive, and there remains a need for larger and more rigorously conducted trials. Overall, soluble fibre is simple to use, inexpensive and safe; however, patients should be reminded to increase their intake slowly to avoid exacerbating symptoms. Bran should not be recommended.

\section{Probiotics}

Some investigators have demonstrated that the faecal microbiome of patients with IBS differs significantly from that of healthy volunteers, ${ }^{36}$ and this might, in part, be responsible for causing symptoms, either directly, or via effects on gastrointestinal transit. ${ }^{37} 38$
This has led to interest in whether probiotics, which are live or attenuated microorganisms that may have beneficial effects in humans, can be used to alter the microbiome, and thereby improve symptoms.

The results of a systematic review and meta-analysis of 53 RCTs of probiotics, involving 5545 patients, showed that combination probiotics, evaluated in $21 \mathrm{RCTs}$, had a significant effect $(\mathrm{RR}=0.79 ; 95 \% \mathrm{CI}$ 0.68 to 0.91$).{ }^{39}$ A total of 33 RCTs reported effect of probiotics on either global IBS symptoms and abdominal pain. Once again, combination probiotics showed a significant benefit over placebo in this analysis; however, for single-organism probiotics containing either Lactobacillus or Bifidobacterium alone, no benefit was observed. With respect to effect on bloating, combination probiotics showed a non-significant trend towards a reduction in bloating scores, but there was no evidence of benefit with Lactobacillus, Bifidobacterium or Saccharomyces.

On balance, these results suggest some probiotics may be beneficial in IBS; however, which combination, strain or species should be preferred in any individual patient remains unclear. The longer-term efficacy of probiotics is unknown, and the mechanism by which they may work, and their effect on the microbiome, requires clarification. The quality of evidence is also low as the majority of trials are small, and many are at an unclear risk of bias. Overall, it is reasonable to advise patients wishing to try probiotics to take a combination product for up to 12 weeks, but to discontinue treatment if they fail to experience symptomatic improvement.

\section{Exercise}

It is widely accepted that physical exercise plays an important role in maintaining good physical and mental health, ${ }^{40-42}$ and that benefit is derived from even small increases in physical activity. ${ }^{42}$ With respect to gastrointestinal symptoms, exercise can accelerate gastrointestinal transit, ${ }^{43}$ improve intestinal gas clearance in patients with bloating ${ }^{44}$ and might increase gut microbial diversity, with the potential to positively impact symptoms via the gut-brain axis. ${ }^{45}$ It is therefore reasonable to assume that exercise will benefit patients with IBS.

One RCT, comparing 12 weeks of an exercise intervention with usual care, invited 305 patients with IBS to participate, of whom only $56(18 \%)$ agreed. ${ }^{46}$ The exercise group reported significant improvements in constipation, compared with patients assigned to usual care, but there were no significant improvements in other IBS symptoms, or quality of life. In a second trial, 102 patients with IBS were randomised to a physical exercise programme or usual care for 12 weeks, 75 of whom completed the trial. ${ }^{47}$ There was a significant difference in improvement in IBS symptom severity scores with exercise $(p=0.003)$. These positive effects 
persisted in 39 patients followed up for a median of 5.2 years. $^{48}$

A systematic review from 2018 summarised findings from 14 RCTs of exercise therapy in IBS, involving a total of 683 patients, ${ }^{49}$ and included the two aforementioned RCTs. ${ }^{46} 47$ Other interventions studied were diverse, including aerobic exercise, yoga, Taiji and mountaineering. The authors concluded that exercise appeared to be an effective treatment, but highlighted that studies were at high risk of bias. Moreover, heterogeneity of study design prevented formal metaanalysis. Nevertheless, patients with IBS should be encouraged to increase physical activity, where feasible, as there is the potential for symptom improvement.

\section{Leisure time and relaxation}

NICE guidelines for the treatment of IBS advise encouraging patients to make the most of their leisure time, and to create opportunities for relaxation. ${ }^{50}$ The impact of this advice on symptoms and quality of life is uncertain; however, it has been demonstrated that everyday stress and IBS symptoms are related, ${ }^{51}$ and patients with IBS report greater stress than controls. ${ }^{52}$ Although the relationship between stress and gastrointestinal symptoms may be reciprocal, rather than causal, there remains a clear logic for promoting relaxation among patients with IBS, which may benefit some individuals. The role of formal psychological therapy is discussed in more detail below.

\section{SPECIALISED DIETARY ADVICE}

If first-line dietary advice is ineffective, patients should be referred for assessment by a specialist dietitian. It is important to recognise that, although exclusion diets are commonplace in IBS management, the mechanisms by which they might work remain unclear. Dietetic assessment is key to ensuring that any diet is followed correctly, and that nutritional requirements are not compromised.

\section{Low FODMAP diet}

One of the most widely used diets in IBS is a diet low in fermentableoligosaccharides, disaccharides, monosaccharides and polyols (FODMAPs). ${ }^{53} \mathrm{~A}$ systematic review and meta-analysis published in 2018 identified seven RCTs comparing a low FODMAP diet with various dietary controls, including habitual diet or a high FODMAP diet, involving 397 participants. ${ }^{54}$ Meta-analysis demonstrated a benefit in patients adopting a low FODMAP diet, compared with control $(\mathrm{RR}=0.69 ; 95 \% \mathrm{CI} 0.54$ to 0.88$)$. However, quality of evidence was very low. No trials were at low risk of bias, due primarily to the difficulties of blinding in dietary intervention studies, sample sizes were small, and heterogeneity was significant, driven by the variation in the control interventions used in trials. This means the efficacy of a low FODMAP diet may have been overestimated. Furthermore, trials only examined the initial exclusion phase of the diet, and did not evaluate effects of the managed re-introduction of FODMAP-containing foods according to tolerance, which is recommended longer-term. Overall, the exclusion of foods high in FODMAPs may reduce IBS symptoms, and can be recommended to patients, although there is a need for higher quality evidence to guide management.

\section{FIRST-LINE DRUG TREATMENTS}

If dietary and lifestyle advice are inadequate for improving symptoms, then a number of first-line drug treatments, targeting individual symptoms, should be considered.

\section{Antispasmodics and peppermint oil}

Conventional analgesic drugs, such as paracetamol, non-steroidal anti-inflammatory drugs and opiates are unlikely to relieve pain in IBS, and some have the potential to exacerbate gastrointestinal symptoms. Instead, antispasmodic drugs, including peppermint oil, should be used to ameliorate pain and bloating, based on the theory that dysmotility and gut spasm might be the underlying cause of these symptoms, and that antispasmodics relax gut smooth muscle.

A meta-analysis from 2008 identified 22 studies comparing 12 different antispasmodics with placebo in 1778 patients. ${ }^{55}$ Fewer patients assigned to antispasmodics had persistent symptoms after treatment compared with those taking placebo $(\mathrm{RR}=0.68$; 95\% CI 0.57 to 0.81 ), although heterogeneity between studies was significant. The analysis included a wide range of drugs, including some, such as otilonium, cimetropium and pinaverium that are unavailable in many countries. However, hyoscine is commonly prescribed, and pooled results from three RCTs showed that it was an efficacious treatment $(\mathrm{RR}=0.63 ; 95 \% \mathrm{CI} 0.51$ to 0.78). Conversely, neither mebeverine nor alverine were more efficacious than placebo, although, in both cases, data came from a single small trial. Overall, total adverse events were significantly more common with antispasmodics, particularly dry mouth, blurred vision and dizziness.

Another meta-analysis conducted as part of the American College of Gastroenterology guidelines in $2018,{ }^{56}$ and pooling data from seven RCTs, demonstrated a statistically significant result in favour of peppermint oil compared with placebo $(\mathrm{RR}=0.54$; 95\% CI 0.39 to 0.76 ). However, there was significant heterogeneity between study results, and the overall quality of evidence was low. Total adverse events were no more common with peppermint oil compared with placebo.

More recently, network meta-analysis has facilitated comparison of antispasmodics and peppermint oil with other 'traditional' IBS treatments. ${ }^{35}$ Peppermint oil ranked first, and antispasmodics third, for effect on global IBS symptoms, and peppermint oil third, and 
antispasmodics second, for effect on abdominal pain. However, it should be noted that the overall quality of trial data for antispasmodics was very low, and many trials were conducted prior to the Rome criteria being established, making comparison between individual trials and treatments problematic. It should also be emphasised that trials of peppermint oil used specific formulations, yet many preparations are widely available for sale to the public. Formulations designed for sustained small intestinal relief may be efficacious for example ${ }^{57}$ but those designed for ileocolonic release might not. ${ }^{58}$ It is therefore inappropriate to extrapolate results of the network meta-analysis to all preparations of peppermint oil.

Nevertheless, currently available evidence, although modest, supports the role of antispasmodics, particularly hyoscine, and peppermint oil in treating IBS, and NICE recommends that physicians should consider prescribing them. ${ }^{50}$ The two can be used in combination, if desired.

\section{Antidiarrhoeals}

Patients with IBS with diarrhoea (IBS-D) can be particularly debilitated by loose stools, with urgency and incontinence, ${ }^{59}$ restricting and disrupting daily life ${ }^{60}$ Consequently, many patients use loperamide to control their diarrhoeal symptoms. Although widely used, evidence for its efficacy is lacking. There have been only two small trials in IBS, both conducted over 30 years ago, and involving only 42 patients with either IBS-D, ${ }^{61}$ or mixed stool pattern IBS. ${ }^{62}$ A pooled analysis of data from these trials demonstrated no statistically significant effect of loperamide, compared with placebo on global IBS symptoms,${ }^{56}$ although in the RCTs themselves there were improvements in stool frequency and consistency. Despite the fact that patients frequently report inadequate symptom relief with the drug, ${ }^{63}$ and due in part to a lack of efficacious alternatives, it is likely some patients will continue to use loperamide. Indeed, NICE guidance advocates loperamide as the first choice drug for diarrhoea in IBS ${ }^{50}$ but physicians should be aware that patients may be dissatisfied with this strategy.

\section{Laxatives}

NICE guidelines recommend laxatives should be considered for treating IBS-C, with patients advised on how to adjust the dose according to clinical response. ${ }^{50}$ Lactulose should be avoided as it may cause bloating, but otherwise, which laxatives should be preferred is unclear. Both osmotic and stimulant laxatives are efficacious in chronic constipation. ${ }^{64}$ However, there is little evidence in IBS-C, beyond the findings of two trials of polyethylene glycol (PEG), an osmotic laxative. In the first of these studies, 42 patients with IBS-C were randomised to either PEG or placebo for 30 days. ${ }^{65}$ There was relief of symptoms and an increase in bowel movements in both the treatment and the placebo arms of the trial; however, there was no significant difference between the two. Conversely, in another study, which recruited 139 patients with IBS-C, there was a significant increase in spontaneous bowel movements with PEG, compared with placebo, after 4 weeks. ${ }^{66}$ There was also a trend towards improvements in bloating with PEG, but no evidence of benefit in terms of effect on abdominal pain. Unfortunately, the longterm efficacy of laxatives in IBS, which is important given the chronicity of symptoms, remains unclear. Overall, these limited data suggest that PEG might be efficacious at improving bowel frequency in IBS-C, at least in the short-term, but the impact on global symptoms appears minimal. Nevertheless, use of laxatives, which are widely available and relatively inexpensive, is a reasonable first-line approach, with escalation to second-line drugs reserved for patients who report an unsatisfactory clinical response.

\section{SECOND-LINE DRUG TREATMENTS}

Patients may report inadequate relief of symptoms with first-line treatments, and for patients who are referred to see a gastroenterologist, it is perhaps more likely that this will be the case. In this situation, secondline treatment with central neuromodulators, such as tricyclic antidepressants (TCAs) or selective serotonin reuptake inhibitors (SSRIs), can be used. Again, this approach is endorsed by NICE guidelines. ${ }^{50}$ Their use is underpinned by the central role of the gut-brain axis in IBS pathophysiology. The central nervous system (CNS) and enteric nervous system (ENS) interact with each other in a bidirectional manner. The CNS may alter gut physiology, including motility or visceral sensitivity, for example altering bowel habit or the experience of pain, thereby triggering symptoms. Similarly, changes within the gut, including to the microbiome, can feed back to the brain, via the ENS, with effects on CNS function. Among patients with IBS at baseline, there is a significant increase in anxiety and depression at follow-up. ${ }^{67}$ The converse is also true; individuals with higher levels of anxiety and depression at baseline are significantly more likely to develop IBS subsequently. ${ }^{67}{ }^{68}$ Central neuromodulators might act on pathways between gut and brain to improve IBS symptoms.

A systematic review and meta-analysis from 2019 identified 18 RCTS comparing TCAs or SSRIs with placebo in IBS, recruiting a total of 1127 patients, with a significant benefit in favour of central neuromodulators $(\mathrm{RR}=0.66 ; 95 \% \mathrm{CI} 0.57$ to 0.76$)$. However, there was significant heterogeneity between studies, although only among trials of SSRIs. A subgroup analysis showed an overall benefit in favour of TCAs for abdominal pain, compared with placebo $(\mathrm{RR}=0.59$; $95 \%$ CI 0.42 to 0.83 ). The effect of these drugs on bowel habit is unclear. Most studies did not recruit participants on the basis of stool form, nor did they evaluate specific stool consistency endpoints. Given 
that constipation is a frequently reported side effect of TCAs, these drugs may have a positive impact in IBS-D, but there is no clear evidence for this. Equally, using TCAs to treat abdominal pain in patients with IBS-C may exacerbate constipation. In terms of safety, eight RCTs provided data for total adverse events, with a significantly higher incidence with central neuromodulators (RR of any adverse event $=1.56$; $95 \% \mathrm{CI}$ 1.23 to 1.98$)$.

The relative efficacy of central neuromodulators has been compared with other 'traditional' treatments in a network meta-analysis. ${ }^{35}$ TCAs ranked second for effect on global IBS symptoms and first for effect on abdominal pain, whereas SSRIs ranked fifth for global symptoms and fourth for abdominal pain. However, we must interpret the results of this network meta-analysis cautiously. Quality of the evidence underpinning it was moderate at best, with few trials at low risk of bias, and many were conducted prior to standardised clinical definitions of IBS, and in small numbers of patients.

Overall, the available data supports the use of central neuromodulators for treating IBS, when first-line treatments are ineffective. TCAs should be preferred, and can be commenced at a low dose (eg, $10 \mathrm{mg}$ at night, due to risk of drowsiness). The dose can be increased, depending on symptomatic response, although dose titration beyond $50 \mathrm{mg}$ may lead to higher rates of adverse events. If symptoms do not improve, SSRIs are a reasonable alternative. Although there is no evidence from RCTs to support the use of serotonin norepinephrine reuptake inhibitors, they are beneficial in other chronic painful disorders, ${ }^{69}$ and there are reports of efficacy in some patients with IBS, particularly those with psychological comorbidity. ${ }^{70}$

\section{SECOND-LINE DRUG TREATMENTS TARGETING ABNORMALITIES OF STOOL FORM}

As already discussed, antidiarrhoeals and laxatives can be used in the treatment of abnormal stool form; however, where these prove ineffective, second-line drugs targeting abnormalities in bowel habit are available.

\section{Drugs for constipation}

A number of novel secretagogues have been developed over the last 10 years, although not all are widely available. These share a common general mechanism of action, although the precise pharmacological effects differ between drugs. Broadly, they activate ion channels in epithelial cells of the gut mucosa, increasing electrolyte and fluid content of the intestinal lumen, thereby softening stools and increasing gastrointestinal transit.

One of the first of these drugs to be developed and licensed was lubiprostone, a prostaglandin $\mathrm{E}_{1}$ derivative. It activates chloride type- 2 channels on the apical surface of intestinal enterocytes. The efficacy of lubiprostone $8 \mathrm{mcg}$ twice daily in IBS-C was evaluated in two placebo-controlled trials, in a total of 1171 patients. ${ }^{71}$ In both trials, a significantly greater proportion of patients randomised to lubiprostone reported moderate or significant relief of IBS symptoms; however, nausea was a common adverse event, affecting $8 \%$ of participants.

Linaclotide and plecanatide stimulate the guanylate cyclase-C receptor. In two RCTs conducted in North America, linaclotide $290 \mathrm{mcg}$ once daily was superior to placebo for IBS-C, at 12 weeks in one trial, and 26 weeks in the second. ${ }^{72}$ The primary endpoint used was a composite of improvement in both abdominal pain and stool frequency, as recommended by the Food and Drug Administration (FDA) for IBS treatment trials. Plecanatide, at doses of $3 \mathrm{mg}$ or $6 \mathrm{mg}$ once daily, was superior to placebo in two RCTs, recruiting 2189 patients with IBS-C ${ }^{74}$ although there was no difference in efficacy between the two doses. Perhaps unsurprisingly, the main adverse event reported for both drugs was diarrhoea.

Finally, tenapanor, which inhibits the gastrointestinal sodium-hydrogen exchanger-3, is licensed for the treatment of IBS-C in the USA. A phase III placebocontrolled trial of 12 weeks of tenapanor $50 \mathrm{mg}$ twice daily, in 629 patients, assessed response using the FDA composite endpoint. ${ }^{75}$ The drug was significantly more efficacious than placebo. The main adverse event was diarrhoea; $6.5 \%$ of those taking tenapanor discontinued the drug, compared with $0.7 \%$ of those taking placebo.

The relative efficacy of all of these secretagogues has been examined using network meta-analysis, incorporating the results of 15 RCTs, containing 8462 patients. $^{76}$ Linaclotide $290 \mathrm{mcg}$ once daily ranked first for global IBS symptoms, abdominal pain, improvement in bowel habit and the FDA composite endpoint. However, all drugs were significantly better than placebo, and no treatment was more efficacious than another. Overall, these findings support the use of secretagogues in IBS-C. They may be best placed for patients who report inadequate relief following optimal or maximum tolerated doses of laxatives from different classes. ${ }^{50}$ Patient response should be assessed after 3 months of treatment, and the drug discontinued if this is deemed inadequate.

\section{Drugs for diarrhoea}

A number of second-line drugs with a diverse range of mechanisms of action are available for treating IBS-D. One of these is the minimally absorbed antibiotic rifaximin. The rationale for its use is the observation that patients with IBS can exhibit changes in their faecal microbiota, ${ }^{37}$ and because some studies have shown an overlap between small intestinal bacterial overgrowth and IBS, although evidence for this is largely of low quality. ${ }^{77}$ In two RCTs, each recruiting almost 600 patients, rifaximin $500 \mathrm{mg}$ three times daily for 14 days was superior to placebo. ${ }^{78}$ Efficacy 
was defined as adequate relief of IBS symptoms for two of the first 4 weeks after completion of treatment. However, the difference in response rates between treatment and placebo arms was modest, at around $8 \%$. The main adverse event was headache, affecting $6 \%$ of patients. Due to the modest effect, and concerns over potential for adverse events with repeated courses of rifaximin, FDA approval was not forthcoming. A 're-treatment' trial was therefore conducted. In this study, 2579 patients with IBS-D received a 2-week course of open-label rifaximin. The 636 patients who responded and then relapsed were re-randomised to up to two further 2-week courses of rifaximin, 10 weeks apart, or placebo. ${ }^{79}$ After the first course, 33\% of those taking rifaximin responded compared with $25 \%$ of those taking placebo, with similar response rates following the second course. In each case, these differences reached statistical significance, but were again only modest.

Drugs that activate $\mu$-opioid receptors in the intestine, such as loperamide, retard gut motility and can treat diarrhoea, whereas those acting on $\delta$-opioid receptors can improve pain. Eluxadoline, a mixed $\mu$-opioid and $\delta$-opioid receptor drug, has been evaluated in two RCTs in IBS-D, recruiting over 2400 patients. ${ }^{80}$ The primary endpoint was a composite of improvement in abdominal pain and stool consistency at 12 weeks. Both trials demonstrated that eluxadoline at dose of $75 \mathrm{mg}$ twice daily and $100 \mathrm{mg}$ twice daily were significantly more efficacious than placebo; however, differences in response rates were modest. In a subsequent study, 346 adults with IBS-D who reported inadequate symptom relief with loperamide were randomised to receive eluxadoline $100 \mathrm{mg}$ twice daily or placebo for 12 weeks. ${ }^{81}$ Once again, a significantly greater proportion of patients taking eluxadoline achieved the composite endpoint, compared with those taking placebo. A particular concern with eluxadoline is the risk of pancreatitis, especially in patients with prior cholecystectomy.

5 -hydroxytryptamine- ${ }_{3}\left(5-\mathrm{HT}_{3}\right)$ receptor antagonists, such as alosetron and ramosetron, retard gut motility. A previous meta-analysis of eight RCTs of alosetron for the treatment of IBS-D, involving 4987 patients, demonstrated a benefit of alosetron $(\mathrm{RR}=0.79$; $95 \%$ CI 0.69 to 0.90 ) when compared with placebo. ${ }^{82}$ Although licensed for use in women with IBS-D in the USA, the drug was withdrawn due to subsequent safety concerns relating to ischaemic colitis and severe constipation. It has been reintroduced for the treatment of severe IBS-D in women in the USA, and observational data from around 2000 patients suggest it is safe and efficacious in this patient group, ${ }^{83}$ but it is not available elsewhere. There are no such safety concerns with ramosetron, and data from five Japanese RCTs demonstrate consistently that it is significantly more efficacious than placebo for treating IBS-D. ${ }^{84}$ Ramosetron is only available in Japan and some other Asian countries. However, data from a small crossover trial of ondansetron suggest this $5-\mathrm{HT}_{3}$ receptor antagonist may also be beneficial in IBS-D; ${ }^{85}$ a parallel group RCT is currently underway in the UK. ${ }^{86}$

A network meta-analysis comparing the relative efficacy of many of the above drugs in IBS-D demonstrated all were more efficacious than placebo, but $5-\mathrm{HT}_{3}$ receptor antagonists appeared to be most efficacious. ${ }^{87}$ Alosetron $1 \mathrm{mg}$ twice daily ranked first for global symptoms, stool consistency and the FDArecommended composite endpoint of improvement in both abdominal pain and stool consistency. Ramosetron $2.5 \mathrm{mcg}$ once daily ranked first for abdominal pain. Both these drugs appeared more efficacious than either eluxadoline or rifaximin for some endpoints.

Unfortunately, the availability of second-line drug options for IBS-D is limited in many countries. Rifaximin is licensed in North America for IBS, but is not universally available, and eluxadoline has been withdrawn in many countries. It would appear that $5-\mathrm{HT}_{3}$ receptor antagonists are the most efficacious and, where alosetron or ramosetron are unavailable, ondansetron is a reasonable alternative. Other options include bile acid sequestrants, such as colesevelam, given the overlap between IBS and bile acid diarrhoea, ${ }^{88}$ although there are no RCTs of these agents in IBS-D.

\section{PSYCHOLOGICAL THERAPIES}

The efficacy of a number of psychological therapies in IBS has been investigated. Among the most widely used is cognitive behavioural therapy (CBT). Early trials of CBT suggested it was efficacious in IBS ${ }^{89} 90$ although individual trial results are conflicting, with some RCTs finding no benefit compared with standard IBS care. ${ }^{91}$ One problem with any trial of psychological therapy is the inability to blind participants to treatment, meaning studies are rarely at low risk of bias. Furthermore, sample sizes are often small, reflecting the intensive nature of psychological interventions, which often require a skilled practitioner working face-to-face with a motivated patient over several weeks. These practical constraints may limit availability in clinical practice. More recently, larger studies have examined the role of minimal-contact $\mathrm{CBT},{ }^{92}$ which participants can self-administer at home, or CBT delivered via the telephone or Internet. ${ }^{93}$ These approaches require therapist input, but at a reduced frequency, meaning they can be made more widely available. Results of these trials suggest these approaches are efficacious at improving IBS symptoms. ${ }^{92} 93$ The beneficial effects of CBT delivered over the telephone or via the Internet persisted up to 24 months after completion of treatment in one trial. ${ }^{94}$

Gut-directed hypnotherapy has also been used in IBS, and, again, small studies suggest it is efficacious, ${ }^{95} 96$ although it has been suggested that delivery outside specialist centres is less beneficial. ${ }^{97}$ Similar to 
CBT, treatment with hypnotherapy requires a skilled practitioner, but has been delivered remotely in one uncontrolled study. ${ }^{98}$ Group hypnotherapy may also improve patient access to treatment. In a multicentre RCT comparing individual and group hypnotherapy with educational support as a control, hypnotherapy was significantly more efficacious than education for adequate relief of symptoms at 3 months and, in a per-protocol analysis, group hypnotherapy was noninferior to individual hypnotherapy. ${ }^{99}$

In a network meta-analysis comparing all available psychological therapies with each other that included 41 RCTs, comprising 4072 participants, treatments with the greatest evidence for efficacy, having both the largest number of trials and recruiting the greatest numbers of patients, were self-administered or minimal contact CBT (RR $=0.61 ; 95 \% \mathrm{CI} 0.45$ to 0.83 ), faceto-face CBT (RR $=0.62 ; 95 \% \mathrm{CI} 0.48$ to 0.80$)$ and gut-directed hypnotherapy $(\mathrm{RR}=0.67 ; 95 \% \mathrm{CI} 0.49$ to 0.91$).{ }^{100}$ However, it is important to emphasise no psychological therapy was superior, in terms of efficacy, to any other. When only those trials that recruited patients with refractory symptoms were included, again CBT-based interventions, namely group CBT and minimal contact CBT, and gut-directed hypnotherapy were more efficacious than control interventions.

Overall, several psychological therapies are efficacious in IBS, although it remains difficult to know which should be preferred, and patient access may be limited. CBT-based treatment and gut-directed hypnotherapy have the largest evidence base, and CBT has demonstrated longer-term efficacy. NICE recommends psychological therapies for patients who remain symptomatic following medical treatment, but only after 12 months has elapsed. ${ }^{50}$ There is an argument for earlier deployment of such therapies, especially among patients with evidence of psychological comorbidity at baseline as, given our understanding of the role of the gut-brain axis, this could alter the clinical course of IBS, preventing symptoms from becoming refractory and improving outcomes. This should be a focus for future treatment trials.

\section{CONCLUSIONS}

Once a diagnosis of IBS is made, it is important to start timely treatment. Good communication is central to managing the condition, and there should be a focus on exploring patient beliefs about the condition, and any concerns they may have. Clinicians should provide a clear explanation about the disorder, and the rationale for any investigations, including why further investigation may not be necessary and why test results are normal. Initial management should include simple lifestyle and dietary advice, discussion of the potential role for probiotics and the importance of exercise and making time for leisure activities and relaxation. If these measures are ineffective, referral to a dietitian for consideration of a low FODMAP diet is appropriate. First-line drug therapy includes antispasmodics and peppermint oil for the treatment of abdominal pain. Loperamide and laxatives can be tried for the treatment of diarrhoea or constipation, respectively, although evidence for their efficacy is limited.

If these approaches fail to improve symptoms, second-line treatments should be used. Central neuromodulators are useful for their effects on global IBS symptoms and abdominal pain; TCAs should be preferred. For patients with constipation who fail to respond to laxatives, treatment with linaclotide should be offered. Unfortunately, second-line options for the treatment of diarrhoea are limited in some countries. $5-\mathrm{HT}_{3}$ receptor agonists appear to be the most efficacious, and although alosetron or ramosetron are not widely available, ondansetron may be a reasonable alternative. Patients who fail to respond to medical treatment should be referred for consideration of psychological therapy, if they are amenable to this. CBT and gut-directed hypnotherapy have the largest evidence base, but access to these treatments may be limited.

Twitter Christopher J Black @DrCJBlack

Contributors CJB and ACF drafted the article.

Funding The authors have not declared a specific grant for this research from any funding agency in the public, commercial or not-for-profit sectors.

Competing interests None declared.

Patient consent for publication Not required.

Provenance and peer review Commissioned; externally peer reviewed.

ORCID iDs

Christopher J Black http://orcid.org/0000-0001-5449-3603

Alexander Charles Ford http://orcid.org/0000-0001-6371-4359

\section{REFERENCES}

1 Lovell RM, Ford AC. Global prevalence of and risk factors for irritable bowel syndrome: a meta-analysis. Clin Gastroenterol Hepatol 2012;10:712-21.

2 Palsson OS, Whitehead W, Törnblom H, et al. Prevalence of Rome IV functional bowel disorders among adults in the United States, Canada, and the United Kingdom. Gastroenterology 2020;158:1262-73.

3 Mearin F, Lacy BE, Chang L, et al. Bowel disorders. Gastroenterology 2016.

4 Gralnek IM, Hays RD, Kilbourne A, et al. The impact of irritable bowel syndrome on health-related quality of life. Gastroenterology 2000;119:654-60.

5 Koloski NA, Talley NJ, Boyce PM. Epidemiology and health care seeking in the functional Gi disorders: a populationbased study. Am J Gastroenterol 2002;97:2290-9.

6 Yawn BP, Locke GR, Lydick E, et al. Diagnosis and care of irritable bowel syndrome in a community-based population. Am J Manag Care 2001;7:585-92.

7 Shivaji UN, Ford AC. Prevalence of functional gastrointestinal disorders among consecutive new patient referrals to a gastroenterology clinic. Frontline Gastroenterol 2014;5:26671.

8 Canavan C, West J, Card T. Review article: the economic impact of the irritable bowel syndrome. Aliment Pharmacol Ther 2014;40:1023-34. 
9 Black CJ, Ford AC. Rational investigations in irritable bowel syndrome. Frontline Gastroenterol 2020;11:140-147.

10 Spiegel BMR, Gralnek IM, Bolus R, et al. Is a negative colonoscopy associated with reassurance or improved health-related quality of life in irritable bowel syndrome? Gastrointest Endosc 2005;62:892-9.

11 Lacy BE, Ford AC, Talley NJ. Quality of care and the irritable bowel syndrome: is now the time to set standards? Am J Gastroenterol 2018;113:167-9.

12 Halpert A, Godena E. Irritable bowel syndrome patients' perspectives on their relationships with healthcare providers. Scand J Gastroenterol 2011;46:823-30.

13 Bertram S, Kurland M, Lydick E, et al. The patient's perspective of irritable bowel syndrome. J Fam Pract 2001;50:521-5.

14 Harvey JM, Sibelli A, Chalder T, et al. Desperately seeking a cure: treatment seeking and appraisal in irritable bowel syndrome. Br J Health Psychol 2018;23:561-79.

15 Lacy BE, Weiser K, Noddin L, et al. Irritable bowel syndrome: patients' attitudes, concerns and level of knowledge. Aliment Pharmacol Ther 2007;25:1329-41.

16 Bradley S, Alderson S, Ford AC, et al. General practitioners' perceptions of irritable bowel syndrome: a Q-methodological study. Fam Pract 2018;35:74-9.

17 Casiday RE, Hungin AP, Cornford CS, et al. GPs' explanatory models for irritable bowel syndrome: a mismatch with patient models? Fam. Pract 2009;26:34-9.

18 Dixon-Woods M, Critchley S. Medical and lay views of irritable bowel syndrome. Fam Pract 2000;17:108-13.

19 Zulman DM, Haverfield MC, Shaw JG, et al. Practices to foster physician presence and connection with patients in the clinical encounter. JAMA 2020;323:70-81.

20 Owens DM, Nelson DK, Talley NJ. The irritable bowel syndrome: long-term prognosis and the physician-patient interaction. Ann Intern Med 1995;122:107-12.

21 Ford AC, Forman D, Bailey AG, et al. Irritable bowel syndrome: a 10-yr natural history of symptoms and factors that influence consultation behavior. Am J Gastroenterol 2008;103:1229-39.

22 Böhn L, Störsrud S, Törnblom H, et al. Self-Reported foodrelated gastrointestinal symptoms in IBS are common and associated with more severe symptoms and reduced quality of life. Am J Gastroenterol 2013;108:634-41.

23 Simrén M, Månsson A, Langkilde AM, et al. Food-Related gastrointestinal symptoms in the irritable bowel syndrome. Digestion 2001;63:108-15.

24 Pauls RN, Max JB. Symptoms and dietary practices of irritable bowel syndrome patients compared to controls: results of a USA national survey. Minerva Gastroenterol Dietol 2019;65:1-10.

25 Atkinson W, Sheldon TA, Shaath N, et al. Food elimination based on IgG antibodies in irritable bowel syndrome: a randomised controlled trial. Gut 2004;53:1459-64.

26 Ali A, Weiss TR, McKee D, et al. Efficacy of individualised diets in patients with irritable bowel syndrome: a randomised controlled trial. BMJ Open Gastroenterol 2017;4:e000164.

27 Fritscher-Ravens A, Pflaum T, Mösinger M, et al. Many patients with irritable bowel syndrome have atypical food allergies not associated with immunoglobulin E. Gastroenterology 2019;157:109-18.

28 B. D. A.. Irritable bowel syndrome and diet: food fact sheet, 2019. Available: https://www.bda.uk.com/resource/irritablebowel-syndrome-diet.html

29 Cockerell KM, Watkins ASM, Reeves LB, et al. Effects of linseeds on the symptoms of irritable bowel syndrome: a pilot randomised controlled trial. J Hum Nutr Diet 2012;25:43543.
30 de Vries J, Miller PE, Verbeke K. Effects of cereal fiber on bowel function: a systematic review of intervention trials. World J Gastroenterol 2015;21:8952-63.

31 Müller M, Canfora EE, Blaak EE. Gastrointestinal transit time, glucose homeostasis and metabolic health: modulation by dietary fibers. Nutrients 2018;10:275.

32 Algera J, Colomier E, Simrén M. The dietary management of patients with irritable bowel syndrome: a narrative review of the existing and emerging evidence. Nutrients 2019;11:2162.

33 Moayyedi P, Quigley EMM, Lacy BE, et al. The effect of fiber supplementation on irritable bowel syndrome: a systematic review and meta-analysis. Am J Gastroenterol 2014;109:1367-74.

34 Francis CY, Whorwell PJ. Bran and irritable bowel syndrome: time for reappraisal. Lancet 1994;344:39-40.

35 Black CJ, Yuan Y, Selinger CP, et al. Efficacy of soluble fibre, antispasmodic drugs, and gut-brain neuromodulators in irritable bowel syndrome: a systematic review and network meta-analysis. Lancet Gastroenterol Hepatol 2020;5:117-131.

36 Kassinen A, Krogius-Kurikka L, Mäkivuokko H, et al. The fecal microbiota of irritable bowel syndrome patients differs significantly from that of healthy subjects. Gastroenterology 2007;133:24-33.

37 Tap J, Derrien M, Törnblom H, et al. Identification of an intestinal microbiota signature associated with severity of irritable bowel syndrome. Gastroenterology 2017;152:11123.

38 Jeffery IB, O'Toole PW, Öhman L, et al. An irritable bowel syndrome subtype defined by species-specific alterations in faecal microbiota. Gut 2012;61:997-1006.

39 Ford AC, Harris LA, Lacy BE, et al. Systematic review with meta-analysis: the efficacy of prebiotics, probiotics, synbiotics and antibiotics in irritable bowel syndrome. Aliment Pharmacol Ther 2018;48:1044-60.

40 Schuch FB, Vancampfort D, Firth J, et al. Physical activity and incident depression: a meta-analysis of prospective cohort studies. Am J Psychiatry 2018;175:631-48.

41 McDowell CP, Dishman RK, Gordon BR, et al. Physical activity and anxiety: a systematic review and meta-analysis of prospective cohort studies. Am J Prev Med 2019;57:545-56.

42 Warburton DER, Bredin SSD. Health benefits of physical activity: a systematic review of current systematic reviews. Curr Opin Cardiol 2017;32:541-56.

43 Strid H, Simrén M, Störsrud S, et al. Effect of heavy exercise on gastrointestinal transit in endurance athletes. Scand J Gastroenterol 2011;46:673-7.

44 Villoria A, Serra J, Azpiroz F, et al. Physical activity and intestinal gas clearance in patients with bloating. Am J Gastroenterol 2006;101:2552-7.

45 Dalton A, Mermier C, Zuhl M. Exercise influence on the microbiome-gut-brain axis. Gut Microbes 2019;10:555-68.

46 Daley A, Grimmett C, Roberts L, et al. The effects of exercise upon symptoms and quality of life in patients diagnosed with irritable bowel syndrome: a randomised controlled trial. Int J Sports Med 2008;29:778-82.

47 Johannesson E, Simrén M, Strid H, et al. Physical activity improves symptoms in irritable bowel syndrome: a randomized controlled trial. Am J Gastroenterol 2011;106:915-22.

48 Johannesson E, Ringström G, Abrahamsson H, et al. Intervention to increase physical activity in irritable bowel syndrome shows long-term positive effects. World $J$ Gastroenterol 2015;21:600-8.

49 Zhou C, Zhao E, Li Y, et al. Exercise therapy of patients with irritable bowel syndrome: a systematic review of randomized controlled trials. Neurogastroenterol Motil 2019;31:e13461.

50 Hookway C, Buckner S, Crosland P, et al. Irritable bowel syndrome in adults in primary care: summary of updated NICE guidance. BMJ 2015;350:h701. 
51 Dancey CP, Taghavi M, Fox RJ. The relationship between daily stress and symptoms of irritable bowel: a time-series approach. J Psychosom Res 1998;44:537-45.

52 Blanchard EB, Lackner JM, Jaccard J, et al. The role of stress in symptom exacerbation among IBS patients. J Psychosom Res 2008;64:119-28.

53 Whelan K, Martin LD, Staudacher HM, et al. The low FODMAP diet in the management of irritable bowel syndrome: an evidence-based review of FODMAP restriction, reintroduction and personalisation in clinical practice. J Hum Nutr Diet 2018;31:239-55.

54 Dionne J, Ford AC, Yuan Y, et al. A systematic review and meta-analysis evaluating the efficacy of a gluten-free diet and a low FODMAPs diet in treating symptoms of irritable bowel syndrome. Am J Gastroenterol 2018;113:1290-300.

55 Ford AC, Talley NJ, Spiegel BMR, et al. Effect of fibre, antispasmodics, and peppermint oil in the treatment of irritable bowel syndrome: systematic review and metaanalysis. BMJ 2008;337:a2313.

56 Ford AC, Moayyedi P, Chey WD, et al. American College of gastroenterology monograph on management of irritable bowel syndrome. Am J Gastroenterol 2018;113:1-18.

57 Cash BD, Epstein MS, Shah SM. A novel delivery system of peppermint oil is an effective therapy for irritable bowel syndrome symptoms. Dig Dis Sci 2016;61:560-71.

58 Weerts ZZRM, Masclee AAM, Witteman BJM, et al. Efficacy and safety of peppermint oil in a randomized, double-blind trial of patients with irritable bowel syndrome. Gastroenterology 2020;158:123-36.

59 Atarodi S, Rafieian S, Whorwell PJ. Faecal incontinence-the hidden scourge of irritable bowel syndrome: a cross-sectional study. BMJ Open Gastroenterol 2014;1:e000002.

60 Buono JL, Carson RT, Flores NM. Health-Related quality of life, work productivity, and indirect costs among patients with irritable bowel syndrome with diarrhea. Health Qual Life Outcomes 2017;15:35.

61 Lavö B, Stenstam M, Nielsen AL. Loperamide in treatment of irritable bowel syndrome--a double-blind placebo controlled study. Scand J Gastroenterol Suppl 1987;130:77-80.

62 Hovdenak N. Loperamide treatment of the irritable bowel syndrome. Scand J Gastroenterol Suppl 1987;130:81-4.

63 Lacy BE, Chey WD, Cash BD, et al. Eluxadoline efficacy in IBS-D patients who report prior loperamide use. Am J Gastroenterol 2017;112:924-32.

64 Ford AC, Suares NC. Effect of laxatives and pharmacological therapies in chronic idiopathic constipation: systematic review and meta-analysis. Gut 2011;60:209-18.

65 Awad RA, Camacho S. A randomized, double-blind, placebocontrolled trial of polyethylene glycol effects on fasting and postprandial rectal sensitivity and symptoms in hypersensitive constipation-predominant irritable bowel syndrome. Colorectal Dis 2010;12:1131-8.

66 Chapman RW, Stanghellini V, Geraint M, et al. Randomized clinical trial: macrogol/PEG 3350 plus electrolytes for treatment of patients with constipation associated with irritable bowel syndrome. Am J Gastroenterol 2013;108:1508-15

67 Koloski NA, Jones M, Talley NJ. Evidence that independent gut-to-brain and brain-to-gut pathways operate in the irritable bowel syndrome and functional dyspepsia: a 1-year population-based prospective study. Aliment Pharmacol Ther 2016;44:592-600.

68 Koloski NA, Jones M, Kalantar J, et al. The braingut pathway in functional gastrointestinal disorders is bidirectional: a 12-year prospective population-based study. Gut 2012;61:1284-90.

69 Welsch P, Üçeyler N, Klose P, et al. Serotonin and noradrenaline reuptake inhibitors (SNRIs) for fibromyalgia. Cochrane Database Syst Rev 2018;2:Cd010292.
70 Lewis-Fernández R, Lam P, Lucak S, et al. An open-label pilot study of duloxetine in patients with irritable bowel syndrome and comorbid major depressive disorder. J Clin Psychopharmacol 2016;36:710-5.

71 Drossman DA, Chey WD, Johanson JF, et al. Clinical trial: lubiprostone in patients with constipation-associated irritable bowel syndrome--results of two randomized, placebocontrolled studies. Aliment Pharmacol Ther 2009;29:329-41.

72 Rao S, Lembo AJ, Shiff SJ, et al. A 12-week, randomized, controlled trial with a 4-week randomized withdrawal period to evaluate the efficacy and safety of linaclotide in irritable bowel syndrome with constipation. Am J Gastroenterol 2012;107:1714-24. quiz p.1725.

73 Chey WD, Lembo AJ, Lavins BJ, et al. Linaclotide for irritable bowel syndrome with constipation: a 26-week, randomized, double-blind, placebo-controlled trial to evaluate efficacy and safety. Am J Gastroenterol 2012;107:1702-12.

74 Brenner DM, Fogel R, Dorn SD, et al. Efficacy, safety, and tolerability of plecanatide in patients with irritable bowel syndrome with constipation: results of two phase 3 randomized clinical trials. Am J Gastroenterol 2018;113:73545 .

75 Chey WD, Lembo AJ, Rosenbaum DP. Efficacy of Tenapanor in treating patients with irritable bowel syndrome with constipation: a 12-week, placebo-controlled phase 3 trial (T3MPO-1). Am J Gastroenterol 2020;115:281-93.

76 Black CJ, Burr NE, Quigley EMM, et al. Efficacy of Secretagogues in Patients With Irritable Bowel Syndrome With Constipation: Systematic Review and Network Metaanalysis. Gastroenterology 2018;155:1753-63.

77 Ford AC, Spiegel BMR, Talley NJ, et al. Small intestinal bacterial overgrowth in irritable bowel syndrome: systematic review and meta-analysis. Clin Gastroenterol Hepatol 2009;7:1279-86.

78 Pimentel M, Lembo A, Chey WD, et al. Rifaximin therapy for patients with irritable bowel syndrome without constipation. N Engl J Med 2011;364:22-32.

79 Lembo A, Pimentel M, Rao SS, et al. Repeat treatment with rifaximin is safe and effective in patients with diarrheapredominant irritable bowel syndrome. Gastroenterology 2016;151:1113-21.

80 Lembo AJ, Lacy BE, Zuckerman MJ, et al. Eluxadoline for irritable bowel syndrome with diarrhea. N Engl J Med 2016;374:242-53.

81 Brenner DG, C; Jo E. Efficacy and safety of eluxadoline in IBS-D patients who report inadequate symptom control with prior loperamide use: a phase 4 , multicenter, multinational, randomized, placebo-controlled, double-blinded study (relief). Gastroenterology 2018;113:S254-5.

82 Ford AC, Brandt LJ, Young C, et al. Efficacy of 5-HT3 antagonists and 5-HT4 agonists in irritable bowel syndrome: systematic review and meta-analysis. Am J Gastroenterol 2009;104:1831-43.

83 Olden KW, Chey WD, Shringarpure R, et al. Alosetron versus traditional pharmacotherapy in clinical practice: effects on resource use, health-related quality of life, safety and symptom improvement in women with severe diarrheapredominant irritable bowel syndrome. Curr Med Res Opin 2019;35:461-72.

84 Zheng Y, Yu T, Tang Y, et al. Efficacy and safety of 5 -hydroxytryptamine 3 receptor antagonists in irritable bowel syndrome: a systematic review and meta-analysis of randomized controlled trials. PLoS One 2017;12:e0172846.

85 Garsed K, Chernova J, Hastings M, et al. A randomised trial of ondansetron for the treatment of irritable bowel syndrome with diarrhoea. Gut 2014;63:1617-25.

86 TRITON. Recruiting patients with diarrhoea predominant IBS for a new trial, 2019. Available: https://ctru.leeds.ac.uk/ triton 
87 Black CJ, Burr NE, Camilleri M, et al. Efficacy of pharmacological therapies in patients with IBS with diarrhoea or mixed stool pattern: systematic review and network metaanalysis. Gut 2020;69:74-82.

88 Slattery SA, Niaz O, Aziz Q, et al. Systematic review with meta-analysis: the prevalence of bile acid malabsorption in the irritable bowel syndrome with diarrhoea. Aliment Pharmacol Ther 2015;42:3-11.

89 Drossman DA, Toner BB, Whitehead WE, et al. CognitiveBehavioral therapy versus education and desipramine versus placebo for moderate to severe functional bowel disorders. Gastroenterology 2003;125:19-31.

90 Vollmer A, Blanchard EB. Controlled comparison of individual versus group cognitive therapy for irritable bowel syndrome. Behav Ther 1998;29:19-33.

91 Boyce PM, Talley NJ, Balaam B, et al. A randomized controlled trial of cognitive behavior therapy, relaxation training, and routine clinical care for the irritable bowel syndrome. Am J Gastroenterol 2003;98:2209-18.

92 Lackner JM, Jaccard J, Keefer L, et al. Improvement in gastrointestinal symptoms after cognitive behavior therapy for refractory irritable bowel syndrome. Gastroenterology 2018;155:47-57.

93 Everitt HA, Landau S, O'Reilly G, et al. Assessing telephonedelivered cognitive-behavioural therapy (CBT) and webdelivered CBT versus treatment as usual in irritable bowel syndrome (ACTIB): a multicentre randomised trial. Gut 2019;68:1613-23.
94 Everitt HA, Landau S, O'Reilly G, et al. Cognitive behavioural therapy for irritable bowel syndrome: 24-month follow-up of participants in the ACTIB randomised trial. Lancet Gastroenterol Hepatol 2019;4:863-72.

95 Simrén M, Ringström G, Björnsson ES, et al. Treatment with hypnotherapy reduces the sensory and motor component of the gastrocolonic response in irritable bowel syndrome. Psychosom Med 2004;66:233-8.

96 Galovski TE, Blanchard EB. The treatment of irritable bowel syndrome with hypnotherapy. Appl Psychophysiol Biofeedback 1998;23:219-32.

97 Lindfors P, Unge P, Arvidsson P, et al. Effects of gut-directed hypnotherapy on IBS in different clinical settings-results from two randomized, controlled trials. Am J Gastroenterol 2012;107:276-85.

98 Palsson OS, Turner MJ, Whitehead WE. Hypnosis home treatment for irritable bowel syndrome: a pilot study. Int $J$ Clin Exp Hypn 2006;54:85-99.

99 Flik CE, Laan W, Zuithoff NPA, et al. Efficacy of individual and group hypnotherapy in irritable bowel syndrome (IMAGINE): a multicentre randomised controlled trial. Lancet Gastroenterol Hepatol 2019;4:20-31.

100 Black CJ, Thakur ER, Houghton LA, et al. Efficacy of psychological therapies for irritable bowel syndrome: systematic review and network meta-analysis. Gut 2020;69:1441-51. 